\title{
Spectroscopic Redshift of the Gamma-Ray Blazar B2 1215+30 from Ly $\alpha$ Emission
}

\author{
A. Furniss ${ }^{1}$ (D) , G. Worseck ${ }^{2,3}$ (D) M. Fumagalli ${ }^{4}$ (D) C. A. Johnson ${ }^{5}$ (D) D. A. Williams ${ }^{5}$ (D), P. Pontrelli ${ }^{5}$, and J. X. Prochaska ${ }^{6}$ (D) \\ ${ }^{1}$ Department of Physics, CSU East Bay, Hayward, CA 94542, USA; amy.furniss@ csueastbay.com \\ ${ }^{2}$ Max-Planck-Institut für Astronomie, Königstuhl 17, D-69117 Heidelberg, Germany \\ ${ }^{3}$ Institut für Physik und Astronomie, Universität Potsdam, Karl-Liebknecht-Str. 24/25, D-14476 Potsdam, Germany \\ ${ }^{4}$ Institute for Computational Cosmology and Centre for Extragalactic Astronomy, Durham University, South Road, Durham, DH1 3LE, UK \\ ${ }^{5}$ Santa Cruz Institute for Particle Physics and Department of Physics, University of California, Santa Cruz, CA 95064, USA \\ ${ }^{6}$ Department of Astronomy and Astrophysics, UCO/Lick Observatory, University of California, 1156 High Street, Santa Cruz, CA 95064, USA \\ Received 2018 June 30; revised 2018 November 13; accepted 2018 November 18; published 2019 January 7
}

\begin{abstract}
We report on Cosmic Origin Spectrograph observations of the gamma-ray bright blazar B2 1215+30, collected in 2015 November. These observations allow for the confirmation of the source redshift from the detection of a Ly $\alpha$ emission feature at $\lambda \sim 1374 \AA$. The emission feature places the source at a redshift of $z=0.1305 \pm 0.003$, confirming the source's ground-based spectral measurement. The gamma-ray emission of the source is discussed in the context of the source distance, required for the accurate reconstruction of the intrinsic gamma-ray emission taking the absorption by the extragalactic background light into account. The source distance is found to be low enough that the previously reported detection of an exceptional flaring event from B2 $1215+30$ in 2014 cannot be used to investigate opacity-specific spectral and variability characteristics introduced by possible ultra-high-energy cosmic-ray propagation.
\end{abstract}

Key words: galaxies: distances and redshifts - galaxies: individual (B2 1215+30) - gamma rays: galaxies ultraviolet: galaxies

\section{Introduction}

B2 $1215+30$ is a very high energy (VHE; $E \geqslant 100 \mathrm{GeV})$ emitting blazar, discovered by the MAGIC telescopes (Aleksić et al. 2012), and confirmed with measurements of flaring activity from VERITAS (Aliu et al. 2013; Abeysekara et al. 2017). Recently, emission features have been detected in a high signal-to-noise $(\mathrm{S} / \mathrm{N})$ optical spectrum taken with the GTC (Paiano et al. 2017), where the authors measure two emission lines associated with [O II] and [O III], establishing that the source is at a redshift of 0.131. Blazars are a type of active galaxy with a relativistic jet pointed along our line of sight. Notably, extragalactic photons with energies above about $100 \mathrm{GeV}$, such as those from blazars, are absorbed by the extragalactic background light (EBL) photons via pair production (Nikishov 1962), changing the galaxy's observed spectrum as compared to the intrinsic one. The absorption of the blazar's gamma-rays by the EBL is an energy- and redshift-dependent process (see, e.g., Finke et al. 2010; Domínguez et al. 2011; Franceschini \& Rodighiero 2017), making the cosmological distance to VHE blazars of key importance when studying the VHE emission from these galaxies.

B2 $1215+30$ is a BL Lacertae object (Nieppola et al. 2006; Ackermann et al. 2015), the most common type of VHEdetected extragalactic object. These jetted active galaxies challenge firm spectroscopic redshift measurements as the blazar outshines its host galaxy, showing only very weak or no emission/absorption lines, and often lack evidence for a $\mathrm{Ca} \mathrm{H}$ $+\mathrm{K}$ break in their optical spectra (Marcha et al. 1996; Healey et al. 2007). Before the measurement by Paiano et al. (2017), there were multiple references to the source redshift in the

Original content from this work may be used under the terms of the Creative Commons Attribution 3.0 licence. Any further distribution of this work must maintain attribution to the author(s) and the title of the work, journal citation and DOI. literature. Claims of $z=0.13$ by Bade et al. (1998) and Akiyama et al. (2003) provide no information on the lines that were detected and $z=0.2374$ by Lanzetta et al. (1993) and White et al. (2000) show optical spectra that appear featureless. The target was resolved with the Hubble Space Telescope, showing the host to be a massive elliptical galaxy, which suggests that the source is at low redshift (Scarpa et al. 2000). Additionally, an attempt to measure the source distance was made by Ricci et al. (2015), collecting a featureless spectrum with $\mathrm{S} / \mathrm{N}$ of 60 with the KPNO Mayall $4 \mathrm{~m}$ telescope using the R-C spectrograph.

Past HST observations of VHE blazars have provided solid redshift lower limits from the measurement of $\mathrm{Ly} \alpha$ forest absorption features, and strong statistically set upper limits based on nondetection of more distant absorption features (PG 1424+240, Furniss et al. 2013a; 3C 66A, Furniss et al. 2013b; PG 1553+113, Danforth et al. 2010 and S5 0716+70, Danforth et al. 2013). With the motivation to make similar measurements for B2 1215+30, spectroscopic UV observations of the galaxy were collected with the Cosmic Origins Spectrograph (COS) in 2015 November. These observations resulted in the clear detection of a Ly $\alpha$ emission feature, firmly indicating the source to reside at a redshift of $z=0.1305 \pm 0.003$. This measurement confirms the groundbased redshift measurements provided by [O II] and [O III] features, and can be utilized to accurately calculate the intrinsic gamma-ray emission from the blazar.

\section{Observations and Analysis}

\subsection{Ground-based Measurement Attempts}

In order to spectroscopically determine the redshift of $\mathrm{B} 21215+30$, we took high $\mathrm{S} / \mathrm{N}$ observations with two spectrographs. First was a spectrum taken with the Kast Spectrograph on the Shane $3 \mathrm{~m}$ telescope located at the Lick Observatory on UTC 2015 February 23. Kast splits the 
incoming light beam into a "red side" with a wavelength coverage of 5500-7000 $\AA$ and a "blue side" with coverage of 3500-5500 A. The data were reduced following standard techniques with the Low-Redux pipeline. ${ }^{7}$ With an exposure of $5400 \mathrm{~s}$, we achieved an average $\mathrm{S} / \mathrm{N}$ of 115 on the blue side and 250 on the red side. Despite these high $\mathrm{S} / \mathrm{N}$ measurements, we detect no spectral features allowing for the determination of the redshift of this source.

We additionally took a spectrum with the Echellette Spectrograph and Imager (ESI) on the $10 \mathrm{~m}$ Keck II telescope at the W. M. Keck observatory on UTC 2015 April 25. We aimed to detect faint and weak features in the spectrum with the ESI because it has significantly better spectral resolution than Kast. The data were reduced following standard techniques with the ESIRedux Pipeline. ${ }^{8}$ With an exposure time of $1200 \mathrm{~s}$, we achieved an average $\mathrm{S} / \mathrm{N}$ per pixel of 110 over a wavelength range of 4000-10000 $\mathrm{\AA}$. No features leading to the determination of a redshift for B2 1215+30 were found in the resulting spectrum.

B2 $1215+30$ is routinely observed with the F.L. Whipple Observatory $1.2 \mathrm{~m}$ telescope on Mt. Hopkins, AZ, as part of a long-term, multifilter program for dozens of $\mathrm{TeV}$ blazars. Its SDSS $r^{\prime}$-band magnitude within two days of each of the spectroscopic measurements was measured to be within 0.1 mag of the median value 14.44 observed in $246 r^{\prime}$-band exposures taken between 2013 June and 2018 July.

\subsection{Space-based UV Measurement with HST}

B2 1215+30 was observed with the HST COS on 2015 November 25 at $\mathrm{COS}$ detector lifetime position 3 with the gratings $\mathrm{G} 130 \mathrm{M}$ (central wavelength $1291 \AA, \lambda / \Delta \lambda \simeq 16,000$ at $1300 \AA$, exposure time $7368 \mathrm{~s}$ ) and $\mathrm{G} 160 \mathrm{M}$ (central wavelength $1611 \AA, \lambda / \Delta \lambda \simeq 17,000$ at $1600 \AA$, exposure time 10854 s) under Program 13651 (PI: Furniss). The raw data were retrieved from the Mikulski Archive for Space Telescopes (MAST) in 2016 January, and reduced using a combination of the instrument pipeline CALCOS (v. 2.21) and custom software for improved dark current subtraction and coaddition of individual exposures in the Poisson regime (see Worseck et al. 2016). To reject dark current we employed custom pulse height ranges $2-15$ on both detector segments. For boxcar extraction we used rectangular apertures that preserve the spectrophotometric accuracy in the covered wavelength range (G130M: 35 pixels, G160M: 23 pixels). Geocoronal contamination in the G130M spectrum was flagged (N I $\lambda 1200$, H I $\lambda 1216$, O I $\lambda 1302,1304,1306)$. The coadded spectra were rebinned by 3 pixels, resulting in an $\mathrm{S} / \mathrm{N}=10-20$ across the covered wavelength range depending on the instrument sensitivity and the actual exposure time.

\section{HST/COS Spectral Results}

While our ground-based spectra taken with Kast and ESI exhibit featureless power-law continua characteristic of blazars, our new HST/COS spectrum reveals multiple intervening absorption lines as well as a prominent emission line at $\lambda \sim 1374 \AA$ (Figure 1). The majority of the strong absorption lines can be identified as metal transitions arising within the Milky Way, as confirmed by comparing our COS spectrum to

\footnotetext{
7 http://www.ucolick.org/ xavier/LowRedux/

8 https://www2.keck.hawaii.edu/inst/esi/ESIRedux/index.html
}

the Galactic composite of Danforth et al. (2016). Conversely, we interpret the prominent emission line as $\mathrm{Ly} \alpha$ emission arising from the broad line region of the blazar, allowing us to measure the B2 $1215+30$ redshift as $z=0.1305 \pm 0.003$. Here, the uncertainty reflects a velocity shift $800 \mathrm{~km} \mathrm{~s}^{-1}$ of the line for C IV, as discussed in Richards et al. (2002) and Shen et al. (2011). Notably, there may be a (larger) unknown systematic error that is present due to the well-known discrepancies between redshifts derived from UV broad lines and the systemic redshift (Richards et al. 2002).

Our interpretation of this broad emission line as $\operatorname{Ly} \alpha$ at the blazar redshift is further corroborated by additional absorption lines (or lack thereof) in the COS spectrum. First, the presence of an intergalactic medium (IGM) line at $z \sim 0.1165$ revealed by Ly $\alpha$ and (marginal) Ly $\beta$ transitions provides a redshift lower limit consistent with our redshift estimate. Furthermore, absorption lines redward of the blazar Ly $\alpha$ emission can all be identified as being either of Galactic origin or metal lines associated with lower redshift IGM lines. Thus, the lack of IGM Ly $\alpha$ absorption features within a redshift path of $\Delta z \sim 0.34$ corroborates our redshift measurement (see Furniss et al. 2013b).

\section{Discussion}

Confirming the redshift of B2 $1215+30$ as measured by Paiano et al. (2017) with UV spectroscopic measurements of the $\operatorname{Ly} \alpha$ emission is important for the interpretation of the gamma-ray emission observed from the source. The distance to an extragalactic gamma-ray source is necessary to accurately calculate the EBL-absorption-corrected gamma-ray spectrum of the source. It is also of key importance to the interpretation of the observed emission (partially) resulting from cascades initiated by ultra-high-energy cosmic rays (UHECR, $E \geqslant 1 \mathrm{PeV})$. This is a plausible scenario if AGN are indeed the progenitors of UHECRs, as postulated by, e.g., Szabo \& Protheroe (1994).

If blazars such as B2 $1215+30$ eject sufficiently energetic protons from their ultrarelativistic jet, particle cascades along the extragalactic trajectory of the UHECR will be initiated. As summarized in Essey \& Kusenko (2010), cosmic-ray protons originating from extragalactic sources have a small probability of interacting with the EBL via $p+\gamma_{\mathrm{EBL}} \rightarrow p+\pi^{0}$ and $p+\gamma_{\mathrm{EBL}} \rightarrow n+\pi^{+}$, where the pions produced then quickly decay into photons in the first case or a positron and neutrinos in the second case, all having very high energies.

Since the cosmic-ray protons and resulting cascade particles travel fractions of the extragalactic distance before interaction, any VHE photons resulting from line-of-sight interactions have traveled through a path of lower opacity than directly emitted VHE photons from the source. Moreover, the particles travel at velocities $v<c$, and therefore would carry time-delayed variability characteristics compared to the source. These effects introduce the possibility to observe opacity-dependent spectral and variability characteristics from VHE blazars such as B2 1215+30. Notably, however, these observable affects would be more significant for more distant sources.

The redshift of B2 $1215+30$ places it at a relatively nearby distance, where the theoretical observable effect introduced by UHECR cascades would be indistinguishable from intrinsic emission from the source. We summarize the inability to use VHE gamma-ray spectral and variability measurements of the nearby source B2 1215+30 from Abeysekara et al. (2017) to 


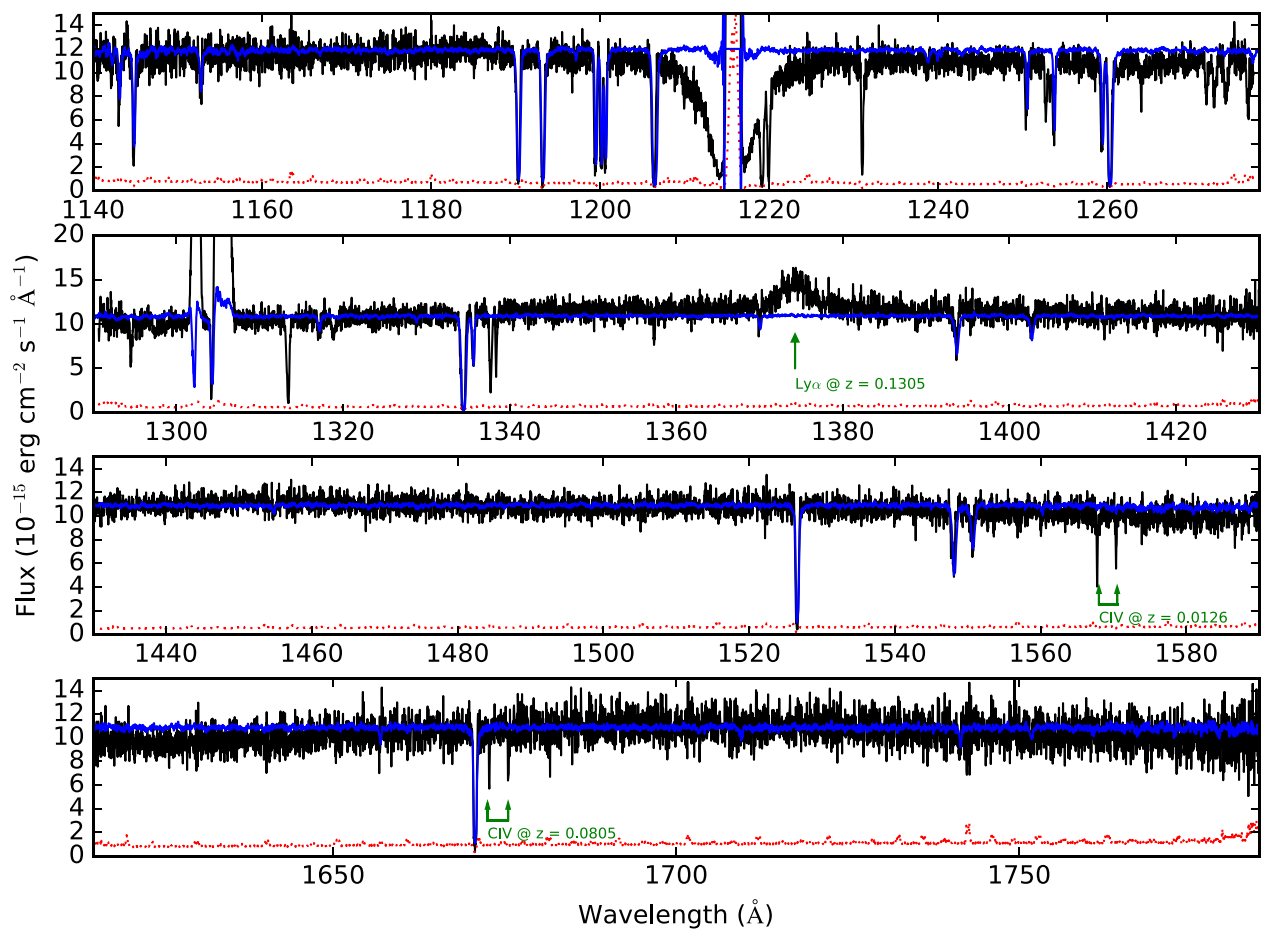

Figure 1. HST/COS spectrum of B2 1215+30 (black solid line), with associated error array (red dotted line). Superimposed, we show in blue the Galactic composite spectrum from Danforth et al. (2016), rescaled to match the continuum level of B2 1215+30. We also label in green (i) the broad emission line identified as Ly $\alpha$ emission at the blazar redshift; (ii) absorption lines redward to the blazar Ly $\alpha$ emission, which we identify as metal lines associated to foreground Ly $\alpha$ forest lines. No additional features within the spectrum, e.g., the unidentified absorption lines around 1230, 1315, or $1340 \AA$, could be associated with the redshift of 0.13 .

disentangle intrinsic gamma-ray emission from the theoretical secondary gamma-ray emission introduced by UHECR interactions along the source line of sight. In 2014 February, VERITAS observed an exceptional flaring event from B2 1215 +30 at VHE. The source flux was then observed to decrease by a factor of more than 30 only $\sim 24$ hours after the observation of the elevated state (Abeysekara et al. 2017). A short 45 minute observation on 2014 February 8 showed the source to display a steady flux of $5.0 \pm 0.1 \times 10^{-10} \mathrm{~cm}^{-2} \mathrm{~s}^{-1}$ above $0.2 \mathrm{TeV}$, dropping to less than $1.4 \times 10^{-11} \mathrm{~cm}^{-2} \mathrm{~s}^{-1}$ above $0.2 \mathrm{TeV}$ on 2014 February 9. Despite high-cloud conditions at the time of observations on 2014 February 8, a spectrum of the source could be reconstructed between 0.2 and $2 \mathrm{TeV}$, with a photon index of $3.1 \pm 0.1_{\text {stat }} \pm 0.6_{\text {sys }}$, in agreement with previous spectral measurements of the source in the VHE band made by Aleksić et al. (2012) and Aliu et al. (2013).

With a confirmed redshift of $z=0.13$, the range of opacity values probed during this flaring event is $\tau=0.2$ at $200 \mathrm{GeV}$ and $\tau=2.1$ at $2 \mathrm{TeV}$ as taken from Finke et al. (2010). Convolved with the $\sim 20 \%$ energy resolution of instruments capable of measuring source emission above $100 \mathrm{GeV}$ (such as VERITAS; Park 2016), this range of opacity values is too narrow to enable a significant study of opacity-specific spectral and variability characteristics of the source, which might be present if B2 1215+30 were ejecting UHECRs. These studies would remain plausible, however, if a more distant source was observed to display a significant flaring event similar to B2 1215+30. For example, a source at a redshift of $z \sim 0.5$ with a similar reconstructed spectral energy range would provide insight into the source spectral and variability patterns spanning opacity values between $\tau=1$ at $200 \mathrm{GeV}$ and $\tau=10$ at $2 \mathrm{TeV}$.
Support for the program HST-GO-13651 was provided by the Space Telescope Institute, which is operated by the Association of Universities for Research in Astronomy, Inc. M.F. acknowledges support from the Science and Technology Facilities Council (grant No. ST/P000541/1). This project has received funding from the European Research Council (ERC) under the European Union's Horizon 2020 research and innovation programme (grant agreement No. 757535). C.A.J., P.P., and D.A.W. acknowledge support from the U.S. National Science Foundation, grants PHY-1307311 and PHY-1707432. We are grateful to Wystan Benbow for obtaining and making available to us data taken with the $1.2 \mathrm{~m}$ telescope at the Fred Lawrence Whipple Observatory.

Facilities: HST/COS, Keck/ESI, Lick/Shane.

\section{ORCID iDs}

A. Furniss (10) https://orcid.org/0000-0003-1614-1273

G. Worseck (iD https://orcid.org/0000-0003-0960-3580

M. Fumagalli (iD https://orcid.org/0000-0001-6676-3842

C. A. Johnson (iD https://orcid.org/0000-0002-0641-7320

D. A. Williams (iD https://orcid.org/0000-0003-2740-9714

J. X. Prochaska (iD https://orcid.org/0000-0002-7738-6875

\section{References}

Abdo, A. A., Ackermann, M., Agudo, I., et al. 2010, ApJ, 716, 30 Abeysekara, A. U., Archambault, S., Archer, A., et al. 2017, ApJ, 836, 205 Ackermann, M., Ajello, M., Atwood, W. B., et al. 2015, ApJ, 810, 14 Akiyama, M., Ueda, Y., Ohta, K., Takahashi, T., \& Yamada, T. 2003, ApJS, 148,275

Aleksić, J., Alvarez, E. A., Antonelli, L. A., et al. 2012, A\&A, 544, A142 Aliu, E., Archambault, S., Arlen, T., et al. 2013, ApJ, 779, 92

Bade, N., Beckmann, V., Douglas, N. G., et al. 1998, A\&A, 334, 459

Danforth, C. W., Keeney, B. A., Stocke, J. T., Shull, J. M., \& Yangsen, Y. 2010, ApJ, 720, 976 
Danforth, C. W., Keeney, B. A., Tilton, E. M., et al. 2016, ApJ, 817, 111

Danforth, C. W., Nalewajko, K., France, K., \& Keeney, B. A. 2013, ApJ, 764, 57

Domínguez, A., Primack, J. R., Rosario, D. J., et al. 2011, MNRAS, 410, 2556

Essey, W., \& Kusenko, A. 2010, APh, 33, 81

Finke, J. D., Razzaque, S., \& Dermer, C. D. 2010, ApJ, 712, 238

Franceschini, A., \& Rodighiero, G. 2017, A\&A, 603, 34

Furniss, A., Fumagalli, M., Danforth, C., Williams, D. A., \& Prochaska, J. X. 2013a, ApJL, 766, 35

Furniss, A., Fumagalli, M., Danforth, C., Williams, D. A., \& Prochaska, J. X. 2013b, ApJ, 766, 35

Healey, S.E., Romani, R. W., Taylor, G. B., et al. 2007, ApJS, 171, 61

Lanzetta, K. M., Turnshek, D. A., \& Sandoval, J. 1993, ApJS, 84, 109

Marcha, M. J. M., Browne, I. W. A., Impey, C. D., \& Smith, P. S. 1996, MNRAS, 281, 425
Nieppola, E., Tornikoski, M., \& Valtaoja, E. 2006, A\&A, 445, 441

Nikishov, A. I. 1962, Sov. Phys. JETP, 14, 393

Paiano, S., Landoni, M., Falomo, R., et al. 2017, ApJ, 837, 144

Park, N. 2016, Proc. ICRC, Bexco, Busan, Korea, 771

Ricci, F., Massaro, F., Landoni, M., et al. 2015, AJ, 149, 160

Richards, G. T., Vanden Berk, D. E., Reichard, T. A., et al. 2002, AJ, 124, 1

Scarpa, R., Urry, C. M., Padovani, P., Calzetti, D., \& Dowd, O. M. 2000, ApJ, 544,258

Shen, Y., Richards, G. T., Strauss, M. A., et al. 2011, ApJS, 194, 45

Szabo, A. P., \& Protheroe, R. J. 1994, APh, 2, 375

White, R. L., Becker, R. H., Gregg, M. D., et al. 2000, ApJS, 126, 133

Worseck, G., Prochaska, J. X., Hennawi, J. F., \& McQuinn, M. 2016, ApJ, 825,144 